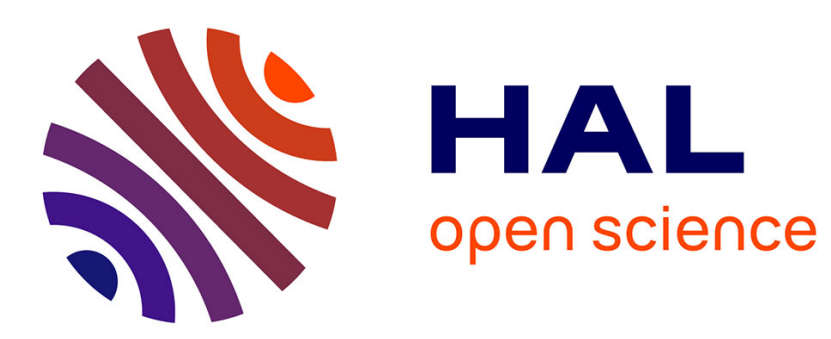

\title{
Solution of Static Field Problems With Random Domains
}

Duy Hung Mac, Stéphane Clenet, Jean-Claude Mipo, Olivier Moreau

\section{To cite this version:}

Duy Hung Mac, Stéphane Clenet, Jean-Claude Mipo, Olivier Moreau. Solution of Static Field Problems With Random Domains. IEEE Transactions on Magnetics, 2010, 46 (8), pp.3385-3388. 10.1109/TMAG.2010.2045358 . hal-00857180

\section{HAL Id: hal-00857180 \\ https://hal.science/hal-00857180}

Submitted on 20 Sep 2013

HAL is a multi-disciplinary open access archive for the deposit and dissemination of scientific research documents, whether they are published or not. The documents may come from teaching and research institutions in France or abroad, or from public or private research centers.
L'archive ouverte pluridisciplinaire HAL, est destinée au dépôt et à la diffusion de documents scientifiques de niveau recherche, publiés ou non, émanant des établissements d'enseignement et de recherche français ou étrangers, des laboratoires publics ou privés. 


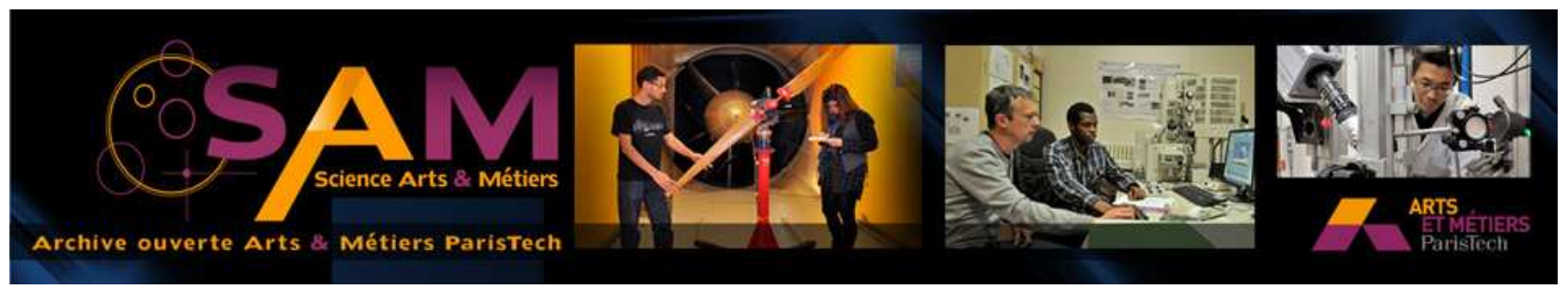

Science Arts \& Métiers (SAM)

is an open access repository that collects the work of Arts et Métiers ParisTech researchers and makes it freely available over the web where possible.

This is an author-deposited version published in: http://sam.ensam.eu

Handle ID: .http://hdl.handle.net/10985/7275

\section{To cite this version :}

Duy Hung MAC, Stéphane CLENET, Jean-Claude MIPO, Olivier MOREAU - Solution of Static Field Problems With Random Domains - IEEE Transactions on Magnetics - Vol. 46, n8, p.3385$3388-2010$ 


\title{
Solution of Static Field Problems with Random Domains
}

\author{
D.H. Mac ${ }^{1,3}$, S. Clénet ${ }^{1,2}$, J.C. Mipo ${ }^{3}$, and O. Moreau ${ }^{4}$ \\ ${ }^{1}$ L2EP/Art et Métiers ParisTech, Lille, 59046 Lille cedex-France \\ ${ }^{2}$ L2EP/LAMEL/Art et Métiers ParisTech, 59046 Lille cedex-France \\ ${ }^{3}$ VALEO-Systèmes Electriques, 94000 Créteil-France \\ ${ }^{4}$ LAMEL/EdF R\&D, 92141 Clamart Cedex-France
}

\begin{abstract}
A method to solve stochastic partial differential equations on random domains consists in using a one to one random mapping function which transforms the random domain into a deterministic domain. With this method, the randomness is then supported by the constitutive relationship of the material. In this paper, this method is applied in electrokinetics in the case of scalar potential and vector potential formulations. An example is treated and the proposed method is compared to a Non Intrusive Method based on the remeshing of the random domains.
\end{abstract}

\section{INTRODUCTION}

The finite element method (FEM) has been widely used to solve the Maxwell equations leading to valuable tools for understanding and predicting the features of electromagnetic devices.

In several cases, the available input data are known with a finite level of confidence. These uncertainties can arise for instance from the aging of the materials or from imperfections of the manufacturing processes. Since the numerical models are more and more accurate due to the improvement of numerical methods (in 3D for example) and also due to the increasing of computer performances, some of these uncertainties can not be considered negligible any more. In several works, a probabilistic approach using random variables is used in order to take into account these uncertainties [1]. In [2], methods to account for uncertainties on the material behavior were used to solve static field problems. However, the case of uncertainties on the geometry is much less studied. In [3], a method to solve differential equations in random domains based on a one to one random mapping function which transforms the random domain into a deterministic one is proposed.

In this paper, we propose to use such approach to solve a static field problem with random dimensions. First, we present the problem to solve in the case of random linear behavior laws. Second, we show how a random domain problem can be transformed into a random behavior law problem using a one to one random mapping. Then, methods, proposed in the literature to treat the case of random material behavior laws, can be applied to solve the problem with random dimension such as the projection method that is shortly presented. Finally, to illustrate the method, a numerical example is presented and a comparison is done with a Non Intrusive Method based on a remeshing procedure.

\section{PROBLEM WITH UNCERTAINTIES ON THE BEHAVIOR LAW}

In this part, we will recall shortly how uncertainties on the behavior law can be taken into account.
The electrokinetics problem with uncertainties on the behavior law defined in deterministic domain $\mathrm{D}$ can be written:

$$
\left\{\begin{array}{c}
\operatorname{div} \mathbf{J}(x, \boldsymbol{\theta})=0 \\
\operatorname{curl} \mathbf{E}(x, \boldsymbol{\theta})=0 \\
\mathbf{J}(x, \boldsymbol{\theta})=\sigma(x, \boldsymbol{\theta}) \cdot \mathbf{E}(x, \boldsymbol{\theta})
\end{array}\right.
$$

The uncertainty on the behavior law is represented by the random field $\sigma(x, \boldsymbol{\theta})$, where $\theta$ is the outcome belonging to the space $\Omega$. The current density $\mathbf{J}$ and the electric field $\mathbf{E}$ are then also random fields.

We assume that the domain $\mathrm{D}$ is bounded by the surface $\Gamma=\Gamma_{1} \cup \Gamma_{2} \cup \Gamma_{3}$ where the boundary conditions are given by:

$$
\left\{\begin{array}{c}
\mathbf{J}(x, \boldsymbol{\theta}) \cdot \mathbf{n}=0 \text { on } \Gamma_{1} \\
\mathbf{E}(x, \boldsymbol{\theta}) \times \mathbf{n}=0 \quad \text { on } \Gamma_{2} \text { and } \Gamma_{3}
\end{array}\right.
$$

where $\mathbf{n}$ is normal unit vector and the electromotive force between $\Gamma_{2}$ and $\Gamma_{3}$ is imposed to $V$. Equation(1) can be solved by either the scalar potential formulation or by the vector potential formulation. If we denote $\varphi(x, \theta)$ the scalar potential that is a random field such that:

$$
\mathbf{E}(x, \theta)=-\operatorname{grad} \varphi(x, \theta)
$$

Equation (1) can be written:

$$
\operatorname{div}(\sigma(x, \theta) \cdot \operatorname{grad} \varphi(x, \theta))=0
$$

The weak formulation becomes:

$$
\int_{D} \operatorname{grad}^{t} \varphi(x, \theta) \cdot \sigma(x, \theta) \cdot \boldsymbol{g r a d} \lambda(x) \cdot \mathrm{d} \Omega(x)=0
$$

where $\lambda(x)$ is a scalar test function that is equal to zero on $\Gamma_{2}$ and $\Gamma_{3}$. In FEM, to approximate the scalar potential, nodal shape functions are commonly used. This problem can be studied using Monte Carlo Simulation Method (MCSM) [4] that is a very reliable method but very time consuming. Alternative methods can also be used that were studied in [1], [2] and [5]: Spectral Stochastic Finite Element Method (SSFEM) and Non-Intrusive Method (NIM). SSFEM and NIM consist in projecting the field $\varphi(x, \theta)$ on space $K(D, \Omega)=S(D) \otimes H(\Omega)$ where $S(D)$ is the space spanned by 
the set of nodal shape functions $\lambda(x)$ (see section II) and $H(\Omega)$ is spanned by a set of orthogonal polynomials $\left\{H_{i}(\theta)\right\}$ (the polynomial chaos) [7]. The main difference between SSFEM and NIM is that with SSFEM, the projection $\varphi(x, \theta)$ is undertaken directly on $K(D, \Omega)=S(D) \otimes H(\Omega)$ while with NIM, $\varphi(x, \theta)$ is firstly projected on $S(D)$ and after on $H(\Omega)$.

\section{PROBLEM WITH UNCERTAINTIES ON GEOMETRY}

With SSFEM, MCSM and NIM, one difficulty in the case of random domains compared to the case of random behavior law is that, a priori, geometric variation leads to a modification of the mesh. Since the boundaries of the domain are random so does the position of the nodes located on that boundary. The shape functions $\lambda(x, \theta)$ associated to these nodes become random fields. The space $S(D)$ is no longer independent of $H(\theta)$, therefore with SSFEM, the random scalar potential $\varphi(x, \theta)$ can not be directly approximated by the projection in $K(D, \Omega)=S(D) \otimes H(\Omega)$. To overcome that difficulty, an idea based on a one to one random mapping function that transforms the random domain to a deterministic domain is proposed in [3]. We will transpose that approach in electrokinetics.

Let consider a domain $\mathrm{D}(\theta)$ with random boundaries and let suppose that it exists a one to one random mapping $X=X(\theta, x)$ which transforms the random domain $\mathrm{D}(\theta)$ to a reference domain $E$ for each outcome $\theta$ (see fig.1).

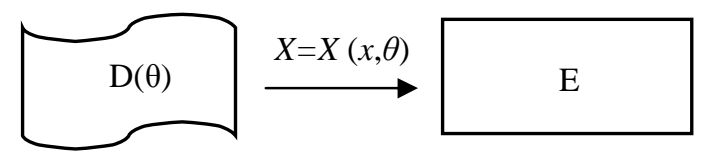

Fig. 1. Transformation method

Thus, applying the random mapping, the weak formulation (5) written on $\mathrm{D}$ can be written on $\mathrm{E}$ and becomes:

$$
\int_{E} \operatorname{grad}^{t} \varphi(X, \theta) \frac{\mathrm{M}^{t}(X, \theta) \cdot \sigma(X) \cdot \mathrm{M}(X, \theta)}{\mid \operatorname{det}(\mathrm{M}(X, \theta) \mid} \operatorname{grad} \lambda(X) \mathrm{d} X=0
$$

where $\mathrm{M}$ is the Jacobian matrix of the mapping. If we denote the conductivity tensor:

$$
\boldsymbol{\sigma}^{\prime}(X, \theta)=\frac{\mathrm{M}^{t}(X, \theta) \cdot \sigma(X) \cdot \mathrm{M}(X, \theta)}{\mid \operatorname{det}(\mathrm{M}(X, \theta) \mid}
$$

the problem with uncertain dimensions on the domain $\mathrm{D}$ can be considered equivalent to a problem with uncertainties on a modified behavior law with a conductivity $\sigma^{\prime}(X, \theta)$ on the reference domain E. In a similar way, the problem (1) can be solved using a vector potential formulation writing $\mathbf{J}=\operatorname{curl} \mathbf{T}$ with $\mathbf{T}$ the vector potential. The weak formulation of the problem is then:

$$
\int_{E} \operatorname{Curl}^{t}(\mathbf{T}(X, \theta)) \cdot \boldsymbol{\sigma}^{-1}(X, \theta) \cdot \boldsymbol{C u r l}(\boldsymbol{\psi}(X)) \cdot \mathrm{d} X=0
$$

with $\psi(\mathrm{X})$ is test function and $\boldsymbol{\sigma}^{,-1}(\mathrm{X}, \theta)$ is inverse matrix of conductivity tensor $\boldsymbol{\sigma}^{\prime}(\mathrm{X}, \theta)$. The vector potential can be approximated in $\mathrm{T}(\mathrm{D}) \otimes H(\Omega)$ with $\mathrm{T}(\mathrm{D})$ the edge element space.

To solve the new problem given by (6) or (8), methods presented in section II can be used. One can note that to use one of these methods, a one to one random mapping function has to be defined.

\section{NUMERICAL APPLICATION}

We focus now on the electrokinetic problem defined in a random domain $\mathrm{D}(\theta)$ presented in Fig. 2. It is a cubic domain $\mathrm{D}_{2}$ with a conductivity $\sigma_{2}=1(\Omega . \mathrm{m})^{-1}$ with an edge length $(2 \mathrm{a}=$ $4 \mathrm{~m})$. This domain holds another cube $D_{1}$ with random dimensions $\left(1_{1}, 1_{2}, 1_{3}\right)$ with a conductivity $\sigma_{1}=10(\Omega . \mathrm{m})^{-1}$.The dimensions $1_{1}(\theta), l_{2}(\theta)$ and $l_{3}(\theta)$ are independent uniform random variables in the interval $[1 ; 1.5](\mathrm{m})$. On two opposite sides of the domain $\mathrm{D}_{2}$ an electromotive force $\mathrm{V}=4$ (Volt) is prescribed. Since the dimensions of $\mathrm{D}_{1}$ are random so does the power. The aim is to calculate the power $\mathrm{W}(\theta)$ dissipated in the domain $\mathrm{D}(\theta)$. The power is approximated by:

$$
\mathrm{W}(\theta)=\sum_{i=1}^{N} w_{i} H_{i}(\theta)
$$

where $\mathrm{H}_{\mathrm{i}}(\theta)$ is the multi-dimensional orthogonal Legendre polynomials and $\mathrm{w}_{\mathrm{i}}$ a real coefficient. To calculate these coefficients, we use a NIM that is based on a projection method [2]. The coefficients are given by:

$$
w_{i}=\frac{\mathrm{E}\left[\mathrm{W}(\theta) \cdot H_{i}(\theta)\right]}{\mathrm{E}\left[H_{i}^{2}(\theta)\right]}
$$

where $\mathrm{E}[\mathrm{X}(\theta)]$ is the expectation of the random variable $\mathrm{X}(\theta)$. The calculation of the denominator can be done analytically whereas the calculation of the numerator can just be done numerically using a quadrature method. For the quadrature method, we consider several specific realizations of the random dimensions $l_{1}(\theta), l_{2}(\theta)$ and $l_{3}(\theta)$. For each realization we solve the problem (6) on the reference domain E applying random mapping that will be describe below. The conductivities on each sub-domain $\mathrm{D}_{1}$ and $\mathrm{D}_{2}$ are reevaluated using (7) for each point of quadrature. The calculation is undertaken on a unique mesh of the reference domain $\mathrm{E}$, only the conductivity distribution changes from a quadrature point to another.

In an opposite way, with a remeshing method (no mapping is required), to each quadrature point which corresponds to a new geometry, the problem (5) is solved with a new mesh.

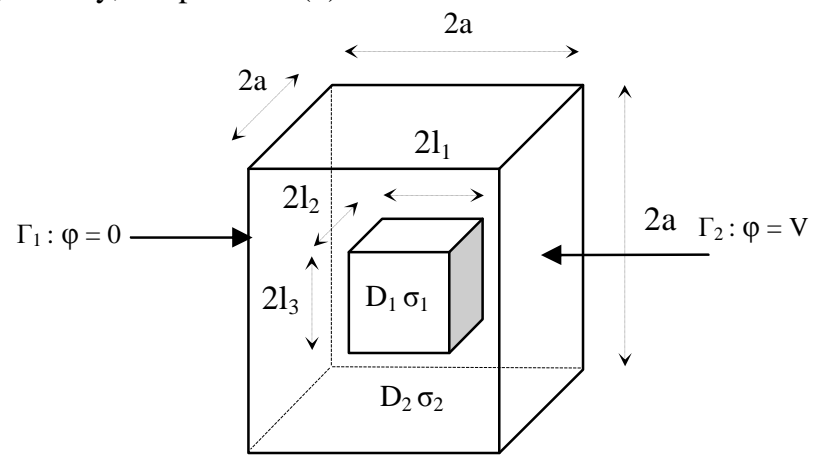

Fig. 2. Electro-kinetic system 
In the following, we will detail the definition of the random mapping. Because of the symmetry of the device, we can divide the domain $\mathrm{D}(\theta)$ into 8 identical sub-domains $\mathrm{D}^{\prime}(\theta)$ where the problem will be studied.

We define the random mapping $\mathrm{X}=\mathrm{X}(\mathrm{x}, \theta)$ that transforms the domain D' into a domain E with $1_{1}=l_{2}=l_{3}=1 \mathrm{~m}$.

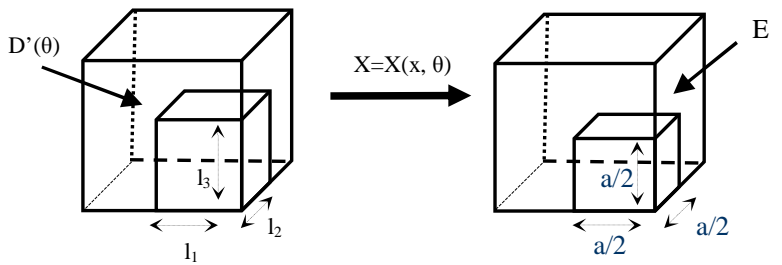

Fig. 3. The transformation $\mathrm{X}$

We divide the domain $\mathrm{D}^{\prime}$ and $\mathrm{E}$ into several sub-domains $D^{\prime}{ }_{i}$ and $E_{i}(8 \geq i \geq 1)$ (Fig. 4). The mapping will be defined for each sub-domain $\mathrm{D}_{\mathrm{i}}$ by a linear transformation (dilation) which is presented in Fig. 5.

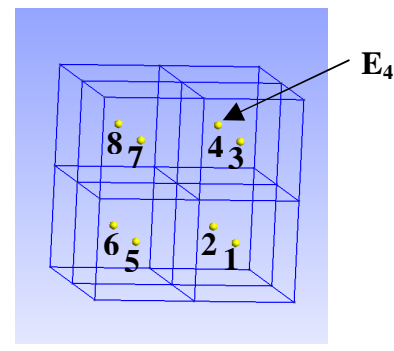

Fig. 4. Division of the random domain $\mathrm{E}$

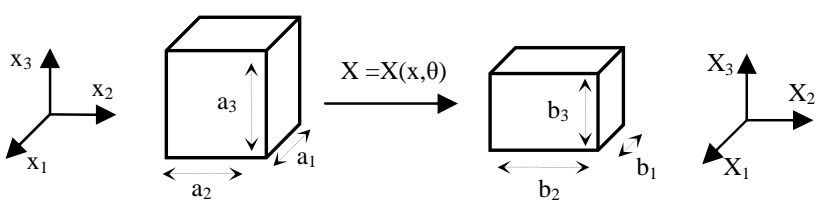

Fig.5. The linear dilation

The linear dilation which transforms a cube of dimensions $a_{1} \times a_{2} \times a_{3}$ into a cube of dimensions $b_{1} \times b_{2} \times b_{3}$ is:

$$
\left[\begin{array}{l}
X_{1} \\
X_{2} \\
X_{3}
\end{array}\right]=\left[\begin{array}{ccc}
\frac{b_{1}}{a_{1}} & 0 & 0 \\
0 & \frac{b_{2}}{a_{2}} & 0 \\
0 & 0 & \frac{b_{3}}{a_{3}}
\end{array}\right]\left[\begin{array}{l}
x_{1} \\
x_{2} \\
x_{3}
\end{array}\right]+C
$$

which $\mathbf{C}$ is a constant vector linking the positions of the two centers of the cubes. We obtain the Jacobian matrix of this transformation:

$$
\mathbf{M}=\left[\begin{array}{ccc}
\frac{b_{1}}{a_{1}} & 0 & 0 \\
0 & \frac{b_{2}}{a_{2}} & 0 \\
0 & 0 & \frac{b_{3}}{a_{3}}
\end{array}\right]
$$

For example, for the transformation of the subdomain $\mathrm{D}_{4}$ into the sub-domain $\mathrm{E}_{4}$ with dimensions $0.5 \mathrm{a} \times 0.5 \mathrm{a} \times 0.5 \mathrm{a}$ we have the following Jacobian matrix (cf Fig. 3):

$$
\mathbf{M}_{4}=\left[\begin{array}{ccc}
\frac{a}{2 \cdot l_{1}} & 0 & 0 \\
0 & \frac{a}{2 \cdot\left(a-l_{2}\right)} & 0 \\
0 & 0 & \frac{a}{2 \cdot\left(a-l_{3}\right)}
\end{array}\right]
$$

Therefore, the equivalent conductivity of this sub-domain can be written under the form:

$$
\sigma_{E_{4}}^{\prime}=\sigma_{2} \cdot\left[\begin{array}{ccc}
\frac{2\left(a-l_{2}\right)\left(a-l_{3}\right)}{a \cdot l_{1}} & 0 & 0 \\
0 & \frac{2 l_{1}\left(a-l_{3}\right)}{a \cdot\left(a-l_{2}\right)} & 0 \\
0 & 0 & \frac{2 l_{1}\left(a-l_{2}\right)}{a \cdot\left(a-l_{3}\right)}
\end{array}\right]
$$

Finally, the initial weak formulation which was written on $\mathrm{D}(\theta)$ under the form:

$$
\sum_{i=1}^{8} \int_{D_{i}^{\prime}} \operatorname{grad}^{t} \varphi(x, \theta) \cdot \sigma_{D_{i}^{\prime}}(x) \cdot \boldsymbol{g r a d} \lambda(x, \theta) \cdot \mathrm{d} x=0
$$

can be written using the mapping on $\mathrm{E}$ :

$$
\sum_{i=1}^{8} \int_{E_{i}} \operatorname{grad}^{t} \varphi(X, \theta) \cdot \boldsymbol{\sigma}_{E_{i}}^{\prime}(X, \theta) \cdot \boldsymbol{g r a d} \lambda(X) \cdot \mathrm{d} X=0
$$

The same approach can be used to solve the vector potential formulation.

For the expression(9), we use an expansion of order 4 of the multivariate Legendre polynomials. A tensorial Legendre Gauss quadrature method is used to calculate the coefficients of the polynomial expansions $\left(4^{3}=64\right.$ points are calculated $)$. The quadrature points are then given by the roots of the Legendre polynomial of order 4 . The domain $\mathrm{E}$ has been meshed with 316 tetrahedra (Fig. 6).

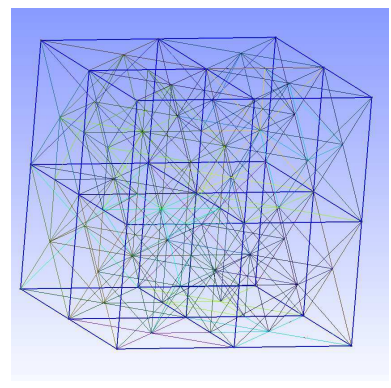

Fig. 6. Mesh of 316 elements

The MCSM has been also used. In that case, a dimensions sample of size $10000\left(1_{1}, l_{2}, l_{3}\right)$ is determined using a random number generator. For each realization, the problem is solved using the FEM coupling with the random mapping. We get at the end a sample of power values of size 10000 . The statistical moments are then estimated. 
The statistical moments obtained with the NIM and the MCSM by using the scalar potential formulation are reported in the Table I. We notice that the NIM gives statistical moments that belong to the $95 \%$ confidence interval obtained with the MCSM. The expansion (9) enables to obtain good results and much faster than the MCSM.

TABLE 1

MONTECARLO SIMULATION METHOD COMPARED WITH NONINTRUSIVE METHOD

\begin{tabular}{|c|c|c|}
\hline \multirow{2}{*}{ Information } & $\begin{array}{c}\text { Monte Carlo method } \\
\text { (95\% confidence interval) }\end{array}$ & Non-intrusive method \\
\cline { 2 - 3 } & Power $(\mathrm{W})$ & Power $(\mathrm{W})$ \\
\hline Mean & {$[28.86: 28.99]$} & 28.91 \\
\hline $\begin{array}{c}\text { Standard } \\
\text { deviation }\end{array}$ & {$[3.34: 3.42]$} & 3.38 \\
\hline Skewness & {$[0.43: 0.56]$} & 0.49 \\
\hline Kurtosis & {$[2.40: 2.69]$} & 2.55 \\
\hline
\end{tabular}

A remeshing method can also be used to approximate the expansion (9) of the power $\mathrm{W}(\theta)$. In fact, for each point of quadrature, a new mesh is generated directly on the domain $\mathrm{D}$ and the problem is solved using both potential formulations. With the mapping method, the geometry and the mesh don't change whereas with the remeshing method, the conductivities in the subdomains don't change. With the mapping method, we use four meshes with $M 1=228, M 2=1729, M 3=2951$, and M4=6825 elements. With the remeshing method, it almost impossible to keep the number of elements constant since the geometry changes from a quadrature point to another but we try to keep this number approximately constant.

Finally, solving the problem with the two methods using both potential formulations leads to four expansions of the power. From each expansion, we can easily determine the mean and the standard deviation. In the Fig. 7 and Fig. 8, we give for the different meshes $(\mathrm{Mi}, \mathrm{i}=1$ to 4$)$, the evolution of the mean and of the standard deviation. First we can see that the scalar potential formulation gives a mean of the power greater than the one given by the vector potential formulation. This property can be deduced from the energy bound property observed in the static electromagnetism in the deterministic case [6]. The exact solution is in between the results given by both formulations. The smaller the gap is between both potential formulations, the more accurate the numerical model. We can see that the gap is a decreasing function of the number of elements and also that the gap is smaller with the mapping method than with the remeshing method. This remark can also be done in the case of the standard deviation.

Since the mesh is modified from a quadrature point to another with the remeshing method, it appears an additional noise on the output of the model (the power $\mathrm{W}(\theta)$ ). This additional noise increases the numerical error. Moreover, the mapping method is less time consuming since there is no need to remesh.

\section{CONCLUSION}

We have presented a method to solve a static field problem with uncertainties on the geometric dimensions. This method consists in using a random mapping that enables to transfer the randomness on the behavior law. We compare this method on an academic example with a method that consists in remeshing the domain. The results obtained show that the mapping method seems to be more accurate and less time consuming. The key point of the mapping method is the determination of the transformation that can't always be determined easily. Methods to determine these mappings are proposed in the literature but need to be compared.

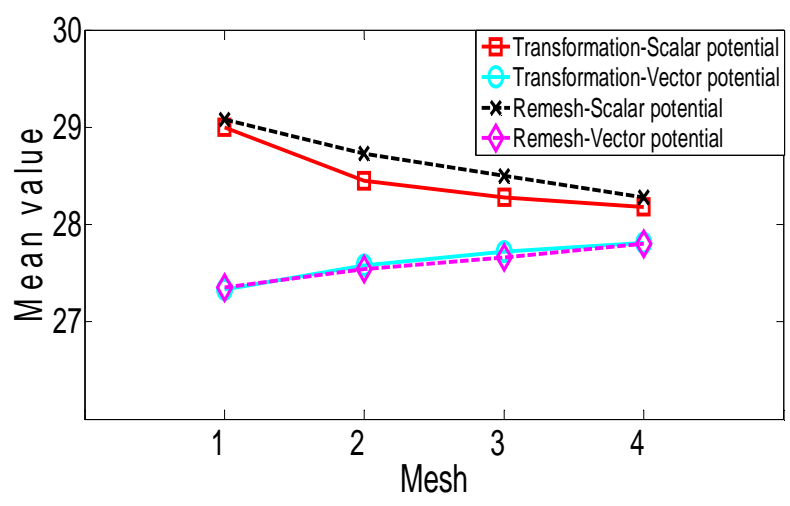

Fig. 7. Mean value of the Power $\mathrm{W}(\theta)$ obtained by different methods

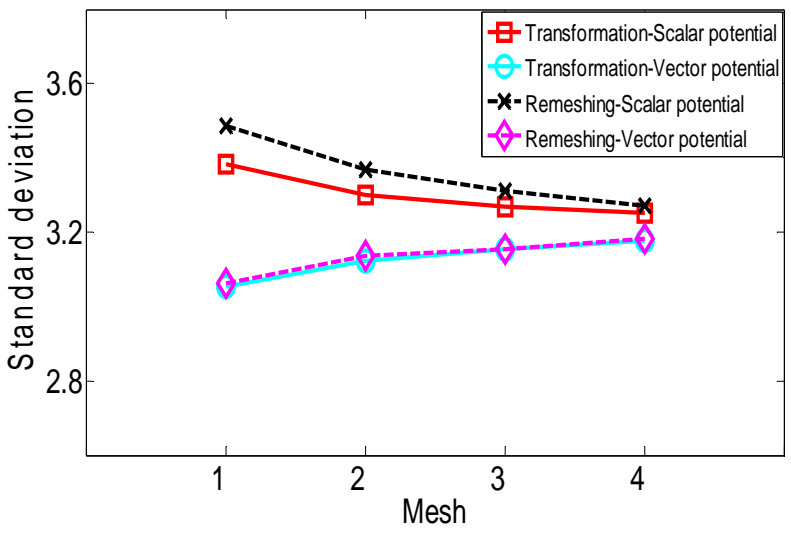

Fig. 8. Standard deviation of the Power $\mathrm{W}(\theta)$ obtained by different methods

\section{REFERENCES}

[1] R. Ghanem, P. D. Spanos, Stochastic Finite Elements: A spectral approach. Mineola, NY: Dover 2003

[2] R. Gaignaire, S. Clenet, O. Moreau, and B. Sudret. 3D spectral stochastic finite element method in electromagnetism. IEEE Trans.Magn. vol.43, no.4, pp. 1209-1212, 2007.

[3] D. Xiu et D. M. Tartakovsky. Numerical methods for differential equations in random domains. SIAM J.SCI COMPUT. No 3, pp.11671185, 2006.

[4] Has' Minsky and Ibragimov, Statistical estimation asymptotic theory. Springer Verlag, 1981

[5] R.Gaignaire, S.Clenet, O.Moreau, B.Sudret. Current Calculation in Electrokinetics Using a Spectral Stochastic Finite Element Method. Magnetics, IEEE Transactions Vol. 44, p.p. 754 - 757, June 2008

[6] W. Prager, J. L. Synge. Approximation in elasticity based on the concept of functions space, Quart. Appl. Math. 5, p.p. 261-269, 1947

[7] D. Xiu and G. Karniadakis, The Wiener Askey polynomial chaos for static differential equations, SIAM J. Sci. Comput. Vol.24, No.2, pp. 619-644. 2002. 